\title{
A Phase Transition in a Quantum Crystal with Asymmetric Potentials*
}

\author{
Alina Kargol and Yuri Kozitsky \\ akargol@golem.umcs.lublin.pl \& jkozi@golem.umcs.lublin.pl \\ Instytut Matematyki, \\ Uniwersytet Marii Curie-Skłodowskiej, \\ Lublin 20-031 Poland
}

December 30, 2017

\begin{abstract}
A translation invariant system of interacting quantum anharmonic oscillators indexed by the elements of a simple cubic lattice $\mathbb{Z}^{d}$ is considered. The anharmonic potential is of general type, which in particular means that it might have no symmetry. For this system, we prove that the global polarization (obtained in the thermodynamic limit) gets discontinuous at a certain value of the external field provided $d \geq 3$, and the particle mass as well as the interaction intensity are big enough. The proof is based on the representation of local Gibbs states in terms of path measures and thereby on the use of the infrared estimates and the Garsia-RodemichRumsey inequality.
\end{abstract}

\section{Introduction and Setup}

The theory of phase transitions in quantum systems has essential peculiarities, which distinguish it from the corresponding theory of classical systems. In this context, it suffices to mention that the existence of phase transitions in the three-dimensional isotropic quantum Heisenberg model has not been proven yet. For lattice models, most of the results in this domain were obtained by means of quantum versions of the method of infrared bounds developed in [9]. The first publication in which the infrared estimates were applied to quantum spin models seems to be the article [8]. After certain modifications this method

\footnotetext{
*Supported by the DFG through the Project 436 POL 113/115/0-1
} 
was applied to a number of models with unbounded Hamiltonians [7, 12, 5, 10], the main characteristic feature of which was the $Z_{2}$-symmetry broken by the phase transition. This symmetry allowed for obtaining an estimate crucial for the method. However in classical models, for proving phase transitions by means of the infrared estimates, symmetry was not especially important, see Theorem 3.5 in [9] and the discussion preceding this theorem. There might be two explanations of such a discrepancy: (a) the symmetry was the key element, but only of the methods employed therein, and, like in the classical case, its lack does not imply the lack of phase transitions; (b) the symmetry is crucial in view of e.g. quantum effects, which stabilize the system, see [2, 3. So far, there has been no possibility to check which of these explanations is true. In this letter, we prove that the system of interacting quantum anharmonic oscillators without any symmetry undergoes a phase transition if in particular the oscillator mass is sufficiently big and hence quantum effects are not so strong. As a consequence, the dilemma mentioned above has been solved in favor of explanation (a).

An infinite system of interacting quantum anharmonic oscillators indexed by the elements of a crystal lattice is called (with a certain abuse of terminology) a quantum anharmonic crystal. Mostly such models are related with ionic crystals containing localized light particles oscillating in the field created by heavy ionic complexes, see [16. An example here can be a KDP-type ferroelectric with hydrogen bounds, in which the particles are protons or deuterons performing one-dimensional oscillations along the bounds. To models of this kind the method of infrared estimates was first applied in 7, 12, where the anharmonic potential was $Z_{2}$-symmetric and of $\phi^{4}$ type. These two properties allowed for obtaining the crucial estimate by means of a purely quantum tool - the Bogoliubov inequality. Afterwards in [5, 10, the method of infrared estimates was extended to cover the case of $Z_{2}$-symmetric anharmonic potentials of general type, which have two deep enough wells. This was achieved my means of a representation of the Gibbs states in the form of imaginary-time Feynman path integrals. The approach in quantum statistical physics based on such a representation is called Euclidean due to its conceptual similarity with the corresponding approach in quantum field theory, see [13. In this approach, the model is treated as a system of interacting classical spins, which are infinitedimensional and unbounded. Thereby, the method of [9] can be applied directly if the estimate mentioned above is obtained.

In this letter, we prove the existence of phase transitions in quantum anharmonic crystals with possibly asymmetric anharmonic potentials. We employ an updated version [1, 4, 11] of the Euclidean method used in [5, 10, combined with some new techniques. Namely, we prove an analog of Lemma 3.4 of [9] by means of the Garsia-Rodemich-Rumsey lemma, which then is used to prove an analog of Theorem 3.5 of [9].

The heuristic Hamiltonian of the model we consider is

$$
H=-\frac{J}{2} \sum_{\ell, \ell^{\prime}:\left|\ell-\ell^{\prime}\right|=1} q_{\ell} q_{\ell^{\prime}}+\sum_{\ell} H_{\ell},
$$


where the sums run through a lattice $\mathbb{L}=\mathbb{Z}^{d}$ and the displacement $q_{\ell}$ is onedimensional. The interaction term is of dipole-dipole type; we assume that $J>0$. The Hamiltonian

$$
H_{\ell}=H_{\ell}^{\mathrm{har}}+V\left(q_{\ell}\right) \stackrel{\text { def }}{=} \frac{1}{2 m} p_{\ell}^{2}+\frac{a}{2} q_{\ell}^{2}+V\left(q_{\ell}\right), \quad a>0,
$$

describes an isolated anharmonic oscillator of mass $m$ and momentum $p_{\ell}$, whereas $H_{\ell}^{\text {har }}$ corresponds to a quantum harmonic oscillator of rigidity $a$. Regarding the anharmonic potential, we assume that it contains an external field $h \in \mathbb{R}$ and is of the form

$$
V(x)=V_{0}(x)-h x,
$$

where $V_{0}$ is continuous, such that $V_{0}(0)=0$, and for all $x \in \mathbb{R}$,

$$
A_{V} x^{2 r}+B_{V} \leq V_{0}(x),
$$

with certain $r>1, A_{V}>0, B_{V} \in \mathbb{R}$. Like all objects of this kind, the Hamiltonian (1) is 'represented' by local Hamiltonians corresponding to nonvoid finite subsets $\Lambda \subset \mathbb{L}$. For such $\Lambda$, we write $\Lambda \Subset \mathbb{L}$; by $|\Lambda|$ we denote the number of elements. The adjective local will always mean the property of being related with a certain $\Lambda \Subset \mathbb{L}$, whereas global will refer to the whole lattice. By $(\cdot, \cdot)$ and $|\cdot|$ we denote the scalar product and norm in $\mathbb{R}^{d}$.

The set $\{\Lambda\}_{\Lambda \subseteq \mathbb{L}}$ is countable; it is a net with the order defined by inclusion. A linearly ordered sequence of subsets $\Lambda \Subset \mathbb{L}$, which exhausts the lattice $\mathbb{L}$, will be called a cofinal sequence. The limit of a sequence of appropriate $A_{\Lambda}$ taken along a cofinal sequence $\mathcal{L}$ will be denoted by $\lim _{\mathcal{L}} A_{\Lambda}$; we write $\lim _{\Lambda} \nearrow_{\mathbb{L}} A_{\Lambda}$ if the limit is taken along $\{\Lambda\}_{\Lambda \Subset \mathbb{L}}$. The same notations will be used for limsup and liminf.

Thereby, the local Hamiltonian is

$$
H_{\Lambda}=-\frac{J}{2} \sum_{\ell, \ell^{\prime} \in \Lambda:\left|\ell-\ell^{\prime}\right|=1} q_{\ell} q_{\ell^{\prime}}+\sum_{\ell \in \Lambda}\left[H_{\ell}^{\mathrm{har}}+V\left(q_{\ell}\right)\right] .
$$

A special kind of $\Lambda \Subset \mathbb{L}$ is the box

$$
\Lambda=(-L, L]^{d} \bigcap \mathbb{L}, \quad L \in \mathbb{N},
$$

which can be turned into a torus by setting periodic conditions on its boundary. The same can be done by equipping $\Lambda$ with the periodic distance $\left|\ell-\ell^{\prime}\right|_{\Lambda}$, the definition of which is standard. By $\mathcal{L}_{\text {box }}$ we denote the set of all boxes. For a box $\Lambda$, we set

$$
H_{\Lambda}^{\mathrm{per}}=-\frac{J}{2} \sum_{\ell, \ell^{\prime} \in \Lambda:\left|\ell-\ell^{\prime}\right|_{\Lambda}=1} q_{\ell} q_{\ell^{\prime}}+\sum_{\ell \in \Lambda}\left[H_{\ell}^{\mathrm{har}}+V\left(q_{\ell}\right)\right],
$$

that is the periodic local Hamiltonian invariant with respect to the translations of the torus $\Lambda$. In the sequel, by writing expressions like $H_{\Lambda}^{\text {per }}$ we tacitely assume 
that $\Lambda$ is a box. Due to (4), both $H_{\Lambda}$ and $H_{\Lambda}^{\text {per }}$ are self-adjoint operators in the Hilbert space $\mathcal{H}_{\Lambda}=L^{2}\left(\mathbb{R}^{|\Lambda|}\right)$, such that for every $\beta>0$,

$$
\begin{array}{ccl}
Z_{\Lambda} & \stackrel{\text { def }}{=} & \operatorname{trace}\left[\exp \left(-\beta H_{\Lambda}\right)\right]<\infty, \\
Z_{\Lambda}^{\text {per }} & \stackrel{\text { def }}{=} & \operatorname{trace}\left[\exp \left(-\beta H_{\Lambda}^{\text {per }}\right)\right]<\infty .
\end{array}
$$

Since the inverse temperature plays no role in our constructions, we set $\beta=1$. We also set $\hbar=1$. Thereby, we define the local Gibbs states

$$
\begin{aligned}
\varrho_{\Lambda}(A) & =\operatorname{trace}\left[A \exp \left(-H_{\Lambda}\right)\right] / Z_{\Lambda}, \quad A \in \mathfrak{C}_{\Lambda}, \\
\varrho_{\Lambda}^{\text {per }}(A) & =\operatorname{trace}\left[A \exp \left(-H_{\Lambda}^{\text {per }}\right)\right] / Z_{\Lambda}^{\text {per }} .
\end{aligned}
$$

Here $\mathfrak{C}_{\Lambda}$ is the local algebra of observables, consisting of all bounded linear operators on $\mathcal{H}_{\Lambda}$. We study the dependence of the averages (9) on $J$, and $h$. Among them are local polarizations

$$
M_{\Lambda}^{\text {per }}(J, h)=\varrho_{\Lambda}^{\text {per }}\left(q_{\ell}\right), \quad M_{\Lambda}(J, h)=\frac{1}{|\Lambda|} \sum_{\ell \in \Lambda} \varrho_{\Lambda}\left(q_{\ell}\right) .
$$

Proposition 1.1. Both $\left\{M_{\Lambda}(j, h)\right\}_{\Lambda \in \mathbb{L}}$ and $\left\{M_{\Lambda}^{\text {per }}(j, h)\right\}_{\Lambda \in \mathcal{L}_{\text {box }}}$ are bounded.

Set

$$
\begin{aligned}
& M_{+}(J, h)=\max \left\{\lim \sup _{\Lambda \nearrow \mathbb{L}} M_{\Lambda}(J, h) ; \lim \sup _{\Lambda \in \mathcal{L}_{\text {box }}} M_{\Lambda}^{\text {per }}(J, h)\right\}, \\
& M_{-}(J, h)=\min \left\{\lim \inf _{\Lambda \nearrow \mathbb{L}} M_{\Lambda}(J, h) ; \lim \inf _{\Lambda \in \mathcal{L}_{\text {box }}} M_{\Lambda}^{\text {per }}(J, h)\right\} .
\end{aligned}
$$

In the following, by a denumerable set we mean the set which is void, finite, or countable.

Proposition 1.2. For every fixed $J$, there exists a denumerable set $\mathcal{R} \subset \mathbb{R}$, such that, for $h \in \mathcal{R}^{c} \stackrel{\text { def }}{=} \mathbb{R} \backslash \mathcal{R}$,

$$
M_{-}(J, h)=M_{+}(J, h) \stackrel{\text { def }}{=} M(J, h) .
$$

The polarization $M(J, h)$, as a function of $h$, is nondecreasing on $\mathcal{R}^{c}$; it is continuous on each its open connected component.

Note that by $\mathcal{R}$ we mean the smallest set with the properties stated.

By Proposition 1.1, it follows that for a specific cofinal sequence $\mathcal{L}$, which may also be composed by boxes, the corresponding sequence of local polarizations has a limit in $\left[M_{-}(J, h), M_{+}(J, h)\right]$. By Proposition [1.2, this interval shrinks into a point if $h \in \mathcal{R}^{c}$, which merely means that, at such $h$, there exists a (global) polarization, independent of the sequence $\mathcal{L}$ along which the thermodynamic limit has been taken. This polarization is continuous on the interval $\left(a_{-}, a_{+}\right) \subset \mathcal{R}^{c}$, where $a_{ \pm}$are two consecutive elements of $\mathcal{R}$. At such $a_{ \pm}$, 
$M(J, h)$ is discontinuous. Therefore, at each $a \in \mathcal{R}$, such that both $(a-\epsilon, a)$, $(a, a+\epsilon)$ are subsets of $\mathcal{R}^{c}$ for a certain $\epsilon>0$, one has

$$
\lim _{h \uparrow a} M(J, h)<\lim _{h \downarrow a} M(J, h) .
$$

At the same time, the set $\mathcal{R}^{c}$ may have empty interior; hence, the global polarization may be nowhere continuous.

Definition 1.3. The model considered undergoes a phase transition (of first order) at certain $J$ and $h_{*}$ if the global polarization, as a function of $h$ at fixed $J$, is discontinuous at this $h_{*}$.

Note that this definition agrees with the known one given by L. Landau and we do not suppose that the phase transition breaks any symmetry.

Theorem 1.4. For every $d \geq 3$, there exist $m_{*}>0$ and $J_{*}>0$ such that for every $m>m_{*}$ and $J>J_{*}$, there exists $h_{*} \in \mathbb{R}$, possibly dependent on $m$ and $J$, such that $M(J, h)$ gets discontinuous at $h_{*}$, i.e., the model undergoes a phase transition.

\section{Proof of the Theorem}

The proof of Theorem 1.4 is based on a number of preparatory statements Propositions and Lemmas. Propositions will be either taken from other sources or discussed in the subsequent parts of the article. Some of them will be proven here. Lemmas are proven in Section 3 .

Define

$$
p_{\Lambda}(J, h)=\log Z_{\Lambda} /|\Lambda| ; \quad p_{\Lambda}^{\text {per }}(J, h)=\log Z_{\Lambda}^{\text {per }} /|\Lambda| .
$$

We shall call these functions the local pressure and the periodic local pressure respectively. Their properties are described by the next two statements. The first one follows directly from the definition (14).

Proposition 2.1. Each $p_{\Lambda}(J, h), p_{\Lambda}^{\mathrm{per}}(J, h)$ is an infinitely differentiable function of $h \in \mathbb{R}$ and $J \in \mathbb{R}$. It is also a convex function of both variables.

One can easily verify that

$$
\frac{\partial}{\partial h} p_{\Lambda}(J, h)=M_{\Lambda}(J, h), \quad \frac{\partial}{\partial h} p_{\Lambda}^{\text {per }}(J, h)=M_{\Lambda}^{\text {per }}(J, h) .
$$

The second statement clarifies the situation with the limiting pressure.

Proposition 2.2. For every $J \geq 0$ and $h \in \mathbb{R}$,

$$
\lim _{\Lambda \nearrow \mathbb{L}} p_{\Lambda}(J, h)=\lim _{\mathcal{L}_{\mathrm{box}}} p_{\Lambda}^{\text {per }}(J, h) \stackrel{\text { def }}{=} p(J, h)
$$


This fact together with those established by Proposition 2.1 yield important information about the global polarizations. It is known, see e.g. [15, pages 34 - 37, that for a convex function $\varphi: \mathbb{R} \rightarrow \mathbb{R}$,

(a) the one-sided derivatives $\varphi_{ \pm}^{\prime}(t)$ exist for every $t \in \mathbb{R}$; the set $\left\{t \in \mathbb{R} \mid \varphi_{+}^{\prime}(t) \neq \varphi_{-}^{\prime}(t)\right\}$ is denumerable;

(b) the point-wise limit $\varphi$ of a sequence of convex functions $\left\{\varphi_{n}\right\}_{n \in \mathbb{N}}$ is a convex function; if $\varphi$ and all $\varphi_{n}$ 's are differentiable at a given $t,\left\{\varphi_{n}^{\prime}(t)\right\}_{n \in \mathbb{N}}$ converges to $\varphi^{\prime}(t)$.

Thereby, the proof of Proposition 1.2 readily follows from the convexity of the pressure and the latter facts.

In the proof of Theorem 1.4, we employ the Duhamel two-point function - a traditional element of the theory of phase transitions in quantum systems, see [8. In our context, it is

$$
D_{\ell \ell^{\prime}}^{\Lambda}=\int_{0}^{1} \int_{0}^{1} \Gamma_{\ell \ell^{\prime}}^{\Lambda}\left(\tau, \tau^{\prime}\right) \mathrm{d} \tau \mathrm{d} \tau^{\prime}=\int_{0}^{1} \Gamma_{\ell \ell^{\prime}}^{\Lambda}(0, \tau) \mathrm{d} \tau, \quad \ell, \ell^{\prime} \in \Lambda
$$

where

$$
\Gamma_{\ell \ell^{\prime}}^{\Lambda}\left(\tau, \tau^{\prime}\right)=\varrho_{\Lambda}^{\mathrm{per}}\left\{q_{\ell} \exp \left[-\left(\tau^{\prime}-\tau\right) H_{\Lambda}^{\mathrm{per}}\right] q_{\ell^{\prime}} \exp \left[\left(\tau^{\prime}-\tau\right) H_{\Lambda}^{\mathrm{per}}\right]\right\}
$$

One can show that

$$
D_{\ell \ell^{\prime}}^{\Lambda} \geq 0,
$$

for all boxes $\Lambda$ and $\ell, \ell^{\prime} \in \Lambda$. By construction, $D_{\ell \ell^{\prime}}^{\Lambda}$ is invariant with respect to the translations of the torus $\Lambda$. Thus, we introduce the set $\Lambda_{*}$ (a Brillouin zone) consisting of

$$
p=\left(p_{1}, \ldots, p_{d}\right), \quad p_{j}=-\pi+\frac{2 \pi}{L} s_{j}, s_{j}=1, \ldots, 2 L, j=1, \ldots, d,
$$

and thereby the Fourier transformation

$$
\begin{aligned}
\widehat{D}_{p}^{\Lambda} & =\sum_{\ell^{\prime} \in \Lambda} D_{\ell \ell^{\prime}}^{\Lambda} \exp \left[\imath\left(p, \ell-\ell^{\prime}\right)\right], \quad p \in \Lambda_{*}, \\
D_{\ell \ell^{\prime}}^{\Lambda} & =\frac{1}{|\Lambda|} \sum_{p \in \Lambda_{*}} \widehat{D}_{p}^{\Lambda} \exp \left[-\imath\left(p, \ell-\ell^{\prime}\right)\right] .
\end{aligned}
$$

Now let us make a more formal definition of the thermodynamic limit. For $\Lambda \subset \Lambda^{\prime} \Subset \mathbb{L}$, one can define the canonical embedding $\mathfrak{C}_{\Lambda} \hookrightarrow \mathfrak{C}_{\Lambda^{\prime}}$, up to which $\mathfrak{C}_{\Lambda}$ be a subalgebra of $\mathfrak{C}_{\Lambda^{\prime}}$. Thereby, we define

$$
\mathfrak{C}^{\mathrm{loc}}=\bigcup_{\Lambda \Subset \mathbb{L}} \mathfrak{C}_{\Lambda}
$$

Equipped with the norm inherited from $\mathfrak{C}_{\Lambda}$, this algebra becomes a normed space. Its completion, $\mathfrak{C}$, is called the algebra of quasi-local observables. 
Proposition 2.3. For every $J>0$ and $h \in \mathbb{R}$, there exists a tending to infinity sequence $\left\{L_{n}\right\}_{n \in \mathbb{N}}$ and hence the sequence $\left\{\Lambda_{n}\right\}_{n \in \mathbb{N}}$ of the corresponding boxes (6)), such that the sequence $\left\{\varrho_{\Lambda_{n}}^{\text {per }}\right\}_{n \in \mathbb{N}}$ converges to a translation invariant state $\varrho^{\text {per }}$ on $\mathfrak{C}$ (called a periodic state) in such a way that, for every polynomial $P\left(q_{\Lambda}\right), \Lambda \Subset \mathbb{L}, q_{\Lambda}=\left(q_{\ell}\right)_{\ell \in \Lambda}$,

$$
\lim _{n \rightarrow+\infty} \varrho_{\Lambda_{n}}^{\text {per }}\left[P\left(q_{\Lambda}\right)\right]=\varrho^{\text {per }}\left[P\left(q_{\Lambda}\right)\right] .
$$

Furthermore, for every $\ell, \ell^{\prime}, \Gamma_{\ell \ell^{\prime}}^{\Lambda_{n}} \rightarrow \Gamma_{\ell \ell^{\prime}}$, uniformly on $\tau, \tau^{\prime} \in[0,1]$.

Note that by (21), one has, c.f. (11),

$$
\varrho^{\text {per }}\left(q_{\ell}\right)=\lim _{n \rightarrow+\infty} M_{\Lambda_{n}}^{\text {per }}(J, h) .
$$

Set, c.f. (17),

$$
D_{\ell \ell^{\prime}}=\int_{0}^{1} \Gamma_{\ell \ell^{\prime}}(0, \tau) \mathrm{d} \tau
$$

By construction, $\Gamma_{\ell \ell^{\prime}}$, and hence $D_{\ell \ell^{\prime}}$, are invariant with respect to the translations of $\mathbb{L}=\mathbb{Z}^{d}$. Thus, one can define

$$
\begin{aligned}
\widehat{D}_{p} & =\sum_{\ell^{\prime}} D_{\ell \ell^{\prime}} \exp \left[\imath\left(p, \ell-\ell^{\prime}\right)\right], \quad p \in(-\pi, \pi]^{d}, \\
D_{\ell \ell^{\prime}} & =\frac{1}{(2 \pi)^{d}} \int_{(\pi, \pi]^{d}} \widehat{D}_{p} \exp \left[-\imath\left(p, \ell-\ell^{\prime}\right)\right] \mathrm{d} p .
\end{aligned}
$$

Proposition 2.4. Suppose that for a given $J>0$, there exists $h_{*}$ such that

$$
\varrho^{\mathrm{per}}\left(q_{\ell}\right)=0
$$

at $h=h_{*}$. Suppose in addition that at $h=h_{*}$ there exists a sequence of boxes $\left\{\Lambda_{n}\right\}_{n \in \mathbb{N}}$ such that

$$
\lim _{n \rightarrow+\infty} \frac{1}{\left|\Lambda_{n}\right|} \sum_{\ell^{\prime} \in \Lambda_{n}} D_{\ell \ell^{\prime}}=\lim _{n \rightarrow+\infty} \frac{1}{\left|\Lambda_{n}\right|^{2}} \sum_{\ell, \ell^{\prime} \in \Lambda_{n}} D_{\ell \ell^{\prime}}>0 .
$$

Then the model undergoes the phase transition at these $J$ and $h_{*}$.

By (24) and (18) it follows from (26) that $\widehat{D}_{p}$ is singular at $p=0$ in this case. On the other hand, by the second line of (24), $\widehat{D}_{p}$ is a distribution; hence, one can write

$$
\widehat{D}_{p}=(2 \pi)^{d} \varkappa \delta(p)+g(p),
$$

where $\delta$ is the Dirac $\delta$-function and $g(p)$ is regular at $p=0$. By (18), $g(p)$, for all $p$, and $\varkappa$ are nonnegative; $\varkappa$ is positive if (26) holds. By (24) and (27),

$$
\varkappa=D_{\ell \ell}-\frac{1}{(2 \pi)^{d}} \int_{(-\pi, \pi]^{d}} g(p) \mathrm{d} p .
$$

Thereby, in order to prove that $\varkappa>0$ one has to estimate $D_{\ell \ell}$ from below and $g(p)$ from above. The latter estimate is obtained in the next statement which can be proven by means of a method used in [8], see Example 4, pages 362 364 . 
Proposition 2.5. Suppose there exists a continuous function $b:(-\pi, \pi]^{d} \backslash$ $\{0\} \rightarrow[0,+\infty)$ satisfying the condition

$$
\int_{(-\pi, \pi]^{d}} b(p) \mathrm{d} p<\infty
$$

and such that for all boxes $\Lambda$,

$$
\widehat{D}_{p}^{\Lambda} \leq b(p), \quad \text { for all } p \in \Lambda_{*} \backslash\{0\} .
$$

Then the function $g$ obeys the estimate

$$
g(p) \leq b(p), \quad \text { for all } p \in(-\pi, \pi]^{d} \backslash\{0\} .
$$

A concrete form of the function $b$ is obtained by the infrared estimates. A detailed presentation of the corresponding method in its application to quantum anharmonic crystals is given in [5, 10, 1, where one can find the following

Proposition 2.6. For every box $\Lambda$, and any $p \in \Lambda_{*} \backslash\{0\}$,

$$
0<\widehat{D}_{p}^{\Lambda} \leq 1 / J E(p),
$$

where

$$
E(p)=\sum_{j=1}^{d}\left[1-\cos p_{j}\right] .
$$

Note that the function $1 / E(p)$ is integrable on $(-\pi, \pi]^{d}$ for $d \geq 3$.

Now we give the statements which finalize the preparation of the proof of the theorem.

Lemma 2.7. For every $m_{0}>0$, there exist $h_{ \pm}\left(m_{0}\right) \in \mathbb{R}, h_{+}\left(m_{0}\right)>h_{-}\left(m_{0}\right)$, such that for all $m>m_{0}$ and $J \geq 0$,

$$
\begin{aligned}
& M_{\Lambda}^{\text {per }}(J, h)>0, \quad \text { for all } h>h_{+}\left(m_{0}\right) \\
& M_{\Lambda}^{\text {per }}(J, h)<0, \quad \text { for all } h<h_{-}\left(m_{0}\right) .
\end{aligned}
$$

The next statement is an analog of Lemma 3.4 of $[9]$.

Lemma 2.8. There exist positive $\varepsilon, \delta$, and $m_{*}$, such that for all $\Lambda$ and $m>m_{*}$,

$$
p_{\Lambda}^{\text {per }}(J, h)-p_{\Lambda}^{\text {per }}(0, h) \geq d(\varepsilon J-\delta) .
$$

Lemma 2.9. Let $m_{*}$ be as above. Then for every $\Lambda, m>m_{*}, J>0$, and $h \in \mathbb{R}$,

$$
\varrho_{\Lambda}^{\text {per }}\left(q_{\ell}^{2}\right) \geq\left[p_{\Lambda}^{\text {per }}(J, h)-p_{\Lambda}^{\text {per }}(0, h)\right] / J d .
$$

One observes that $p_{\Lambda}^{\text {per }}(0, h)$ does not depend on $\Lambda$. By means of Lemmas 2.8, 2.9 we have

$$
\varrho_{\Lambda}^{\mathrm{per}}\left(q_{\ell}^{2}\right) \geq \varepsilon-\delta / J
$$


which can be used to estimate $D_{\ell \ell}$ from below. Note that the lower bound of $\varrho_{\Lambda}^{\text {per }}\left(q_{\ell}^{2}\right)$ was mentioned in the introduction as a crucial estimate; in [7, 12, it was derived by the Bogoliubov inequality.

In our version of the infrared bound method, we estimate the Duhamel function, which is performed by means of (37) and the Bruch-Falk inequality, see Theorem 3.1 in [8] or Theorem IV.7.5, page 392 of [15].

Proposition 2.10 (Bruch-Falk Inequality). It follows that

$$
D_{\ell \ell}^{\Lambda} \geq \varrho_{\Lambda}^{\mathrm{per}}\left(q_{\ell}^{2}\right) \cdot f\left(\frac{1}{4 m \varrho_{\Lambda}^{\mathrm{per}}\left(q_{\ell}^{2}\right)}\right),
$$

where $f:[0,+\infty) \rightarrow[0,1]$ is defined implicitly by

$$
f(\xi \tanh \xi)=\xi^{-1} \tanh \xi, \quad \text { for } \xi>0 ; \quad \text { and } \quad f(0)=1 .
$$

As the right-hand side of (38) is independent of $\Lambda$, one can pass here to the thermodynamic limit along the same sequence of boxes as in (22). Then

$$
D_{\ell \ell} \geq(\varepsilon-\delta / J) \theta(J) ; \quad \theta(J) \stackrel{\text { def }}{=} f\left(J / 4 m_{*}(\varepsilon J-\delta)\right),
$$

where $m_{*}$ is as in Lemma 2.8 and $J>\delta / \varepsilon$.

Proof of Theorem 1.4: If the model has no phase transitions, by Proposition 1.2 the set $\mathcal{R}$ is void and $M(J, h)$ is a continuous function of $h \in \mathbb{R}$ for each $J>0$. This yields, see (12) and Lemma 2.7 that for every $J>0$, there exists $h_{*} \in \mathbb{R}$, such that $M\left(J, h_{*}\right)=0$. On the other hand, by (40) and (32), (31), we have in (28)

$$
\varkappa \geq(\varepsilon-\delta / J) \theta(J)-\frac{1}{J(2 \pi)^{d}} \int_{(-\pi, \pi]^{d}} \frac{\mathrm{d} p}{E(p)} .
$$

As the right-hand side does not depend on $h$, we pick up $J_{*}>0$ such that $\varkappa>0$ for all $J>J_{*}$. For such $J$ and $m>m_{*}$, (26) holds. Then by Lemma 2.4, we get a contradiction with the supposition made at the beginning of the proof.

We conclude this section by giving the path integral representation, 1

$$
\begin{aligned}
M_{\Lambda}^{\mathrm{per}}(J, h) & =\int_{\Omega_{\Lambda}} \omega_{\ell}(0) \mu_{\Lambda}^{\mathrm{per}}\left(\mathrm{d} \omega_{\Lambda}\right), \\
\Gamma_{\ell \ell^{\prime}}^{\Lambda}\left(\tau, \tau^{\prime}\right) & =\int_{\Omega_{\Lambda}} \omega_{\ell}(\tau) \omega_{\ell^{\prime}}\left(\tau^{\prime}\right) \mu_{\Lambda}^{\mathrm{per}}\left(\mathrm{d} \omega_{\Lambda}\right) .
\end{aligned}
$$

Here

$$
\Omega_{\Lambda}=\left\{\omega_{\Lambda}=\left(\omega_{\ell}\right)_{\ell \in \Lambda} \mid \omega_{\ell} \in \mathcal{C}, \text { for all } \ell \in \Lambda\right\},
$$

and $\mathcal{C}$ is the Banach space of all continuous functions $\omega:[0,1] \rightarrow \mathbb{R}$, such that $\omega(0)=\omega(1)$, equipped with the usual supremum norm $|\cdot|_{\mathcal{C}}$. In the Hilbert

\footnotetext{
${ }^{1}$ See [1 4] 11] for more details.
} 
space $\mathcal{L}^{2} \stackrel{\text { def }}{=} L^{2}([0,1], \mathrm{d} \tau)$, one defines the operator $A=-m \mathrm{~d}^{2} / \mathrm{d} \tau^{2}+a$. Its spectrum consists of the eigenvalues

$$
\lambda_{k}=m(2 \pi k / \beta)^{2}+a, \quad k \in \mathbb{Z}
$$

Thus, $A^{-1}$ is of trace class and the Fourier transform

$$
\int_{L_{\beta}^{2}} \exp \left[\imath(\phi, v)_{\mathcal{L}^{2}}\right] \chi(\mathrm{d} v)=\exp \left\{-\frac{1}{2}\left(A^{-1} \phi, \phi\right)_{\mathcal{L}^{2}}\right\}, \phi \in \mathcal{L}^{2}
$$

defines a Gaussian measure $\chi$ on $\mathcal{L}^{2}$, which obviously depends on $m$. By means of (43), one can show that for any $k \in \mathbb{N}$,

$$
\int_{\mathcal{L}^{2}}\left[\omega(\tau)-\omega\left(\tau^{\prime}\right)\right]^{2 k} \chi(d \omega) \leq \frac{2^{k} \Gamma(1 / 2+k)}{m^{k} \Gamma(1 / 2)} \cdot\left|\tau-\tau^{\prime}\right|_{\mathrm{per}}^{k},
$$

which by Kolmogorov's lemma, page 43 of [14], yields that $\chi(\mathcal{C})=1$. Thereby, we redefine $\chi$ as a probability measure on $\mathcal{C}$. An account of its properties may be found in [1]. Thereby, the measure in (41) is obtained with the help of the Feynman-Kac fromula

$$
\mu_{\Lambda}^{\mathrm{per}}\left(\mathrm{d} \omega_{\Lambda}\right)=\exp \left[-I_{\Lambda}^{\mathrm{per}}\left(\omega_{\Lambda}\right)\right] \chi_{\Lambda}\left(\mathrm{d} \omega_{\Lambda}\right) / Z_{\Lambda}^{\mathrm{per}}
$$

as a Gibbs modification of the 'free measure'

$$
\chi_{\Lambda}\left(\mathrm{d} \omega_{\Lambda}\right)=\prod_{\ell \in \Lambda} \chi\left(\mathrm{d} \omega_{\ell}\right) .
$$

Here

$$
I_{\Lambda}^{\mathrm{per}}\left(\omega_{\Lambda}\right)=-\frac{J}{2} \sum_{\ell, \ell^{\prime} \in \Lambda:\left|\ell-\ell^{\prime}\right|_{\Lambda}=1}\left(\omega_{\ell}, \omega_{\ell^{\prime}}\right)_{\mathcal{L}^{2}}+\sum_{\ell \in \Lambda} \int_{0}^{1} V\left(\omega_{\ell}(\tau)\right) \mathrm{d} \tau
$$

\section{Comments and Proof of the Lemmas}

\subsection{Comments on Propositions}

Here we discuss the proof of Propositions 1.1, 2.2, 2.3, 2.4, and 2.6.

By means of the Euclidean realization of the states (14), one can prove that the sets $\left\{\varrho_{\Lambda}\right\}_{\Lambda \Subset \mathbb{L}},\left\{\varrho_{\Lambda}^{\text {per }}\right\}_{\Lambda \in \mathcal{L}_{\text {box }}}$, are relatively compact in the topology which guaranties the convergences stated in Proposition 2.2. The boundedness mentioned in Proposition 1.1 follows from the moment estimates for Euclidean Gibbs measures proven in [4, 11. The existence of periodic Gibbs states was proven in [1]. The estimate (18) follows from the FKG inequality, for the Euclidean Gibbs measures proven in [1, 11]. The proof of (16) was performed in [1], see Lemma 6.4. If (26) holds, then the limiting periodic Euclidean Gibbs states are nonergodic, which certainly means a phase transition, see [11] and the references therein. The estimate (32) is the infrared bound, the proof of which is standard, see [5, 10, 1, 11]. 


\subsection{Proof of Lemma 2.7}

We start by proving the first line in (34). To this end, we find a strictly increasing function $\phi:\left[h_{+}\left(m_{0}\right),+\infty\right) \rightarrow \mathbb{R}$ such that

$$
p_{\Lambda}^{\text {per }}(J, h) \geq \phi(h) \quad \text { for } h \geq h_{+}\left(m_{0}\right) .
$$

Then we use the convexity of $p_{\Lambda}^{\mathrm{per}}(J, \cdot)$ and get the result in question by (15). Let us split the potential $V_{0}$ into even and odd parts

$$
V_{0}(x)=V_{0}^{\mathrm{e}}(x)+V_{0}^{\mathrm{o}}(x) .
$$

Thereby, for $b>0$, we choose $\underline{h}>0$ such that, for all $h>\underline{h}, h x_{\ell}-V_{0}^{\mathrm{e}}\left(x_{\ell}\right)$ is an increasing function of $x_{\ell} \in[-b, b]$. Set, c.f. (42), (46),

$$
\begin{gathered}
\mathcal{C}_{b}=\left\{\left.\omega \in \mathcal{C}|| \omega\right|_{\mathcal{C}} \leq b\right\}, \quad \Omega_{\Lambda}^{b}=\left\{\omega_{\Lambda}=\left(\omega_{\ell}\right)_{\ell \in \Lambda} \mid \omega_{\ell} \in \mathcal{C}_{b}, \quad \ell \in \Lambda\right\}, \\
Z_{\Lambda}^{b}(J, h)=\int_{\Omega_{\Lambda}^{b}} \exp \left[-I_{\Lambda}^{\mathrm{per}}\left(\omega_{\Lambda}\right)\right] \chi_{\Lambda}\left(\mathrm{d} \omega_{\Lambda}\right) .
\end{gathered}
$$

Obviously,

$$
p_{\Lambda}^{\mathrm{per}}(J, h) \geq \frac{1}{|\Lambda|} \log Z_{\Lambda}^{b}(J, h) .
$$

By the first GKS inequality, see e.g., Theorem 12.1 in [14,

$$
Z_{\Lambda}^{b}(J, h) \geq Z_{\Lambda}^{b}(0, h) \stackrel{\text { def }}{=} \exp (|\Lambda| \phi(h)), \quad \text { for all } h>\underline{h} .
$$

Here

$$
\exp [\phi(h)]=\int_{\mathcal{C}_{b}} \exp \left\{\int_{0}^{1}\left[h \omega(\tau)-V_{0}(\omega(\tau))\right] \mathrm{d} \tau\right\} \chi(\mathrm{d} \omega) .
$$

By Jensen's inequality, for every $\tilde{h}>0$ and $h \geq \tilde{h}$,

$$
\phi(h) \geq(h-\tilde{h}) \gamma(m, \tilde{h})+\phi(\tilde{h}), \quad \gamma(m, h) \stackrel{\text { def }}{=} \phi^{\prime}(h) .
$$

By (45), one can show, see 11, that for any $m_{0}>0$, the family of the corresponding measures $\{\chi\}_{\mid m \geq m_{0}}$ is tight as measures on $\mathcal{C}$. On the other hand, the right-hand side of (52) can be extended to the whole complex plane as an entire ridge function of $h$ with the ridge being the real axis. Thereby, for any $m_{0}>0$, there should exist $\tilde{h}$ such that

$$
\gamma_{*}(\tilde{h}) \stackrel{\text { def }}{=} \inf _{m \geq m_{0}} \gamma(h, \tilde{h})>0 .
$$

Then for a fixed $m_{0}$, we take $h_{+}\left(m_{0}\right)=\max \{\tilde{h}, \underline{h}\}$, which yields (48) and hence the first part of (34). Since we have not employed any property of $V_{0}^{\mathrm{e}}$, the rest of the lemma can be proven by changing the sign of $h$ and all $\omega_{\ell}$. 


\subsection{The Main Estimate}

The proof of Lemmas 2.8, 2.9 is based on the estimate which we derive now.

The path measure $\nu_{h}$ corresponding to the anharmonic oscillator (2) with the external field $h$ is defined as a probability measure on $\mathcal{C}$ by the following expression, c.f. (3), (46), and (47),

$$
\nu_{h}(\mathrm{~d} \omega)=\frac{1}{N_{h}} \exp \left[h \int_{0}^{1} \omega(\tau) \mathrm{d} \tau-\int_{0}^{1} V_{0}(\omega(\tau)) \mathrm{d} \tau\right] \chi(\mathrm{d} \omega),
$$

where $1 / N_{h}$ is a normalization factor. For a given fixed $m_{0}$, let $h_{ \pm}\left(m_{0}\right)$ be as in Lemma 2.7 Then for $\epsilon>0$ and

$$
h \in\left[h_{-}\left(m_{0}\right)-\epsilon, h_{+}\left(m_{0}\right)+\epsilon\right], \quad m>m_{0},
$$

by (45) we readily get

$$
\int_{\mathcal{C}}\left[\omega(\tau)-\omega\left(\tau^{\prime}\right)\right]^{2 k} \nu_{h}(d \omega) \leq m^{-k} Q_{k} \cdot\left|\tau-\tau^{\prime}\right|_{\mathrm{per}}^{k}, \quad k \in \mathbb{N},
$$

which holds, uniformly in $h$ and $m$ obeying (55), with $Q_{k}$ depending on $\epsilon$ only. In the sequel, we always assume that $h$ and $m$ are chosen according to (55). Since $V_{0}$ is continuous and defined on the whole $\mathbb{R}$, every finite-dimensional projection of $\nu_{h}$ is non-degenerate, which yields that for every $n \in \mathbb{N}$ and $c>0$ both sets

$$
C^{ \pm}(n ; c) \stackrel{\text { def }}{=}\{\omega \in \mathcal{C} \mid \pm \omega(j / n) \geq c, \quad j=1, \ldots, n\}
$$

are such that $\nu_{h}\left[C^{ \pm}(n ; c)\right]>0$.

Lemma 3.1. For every integer $n \geq 2$ and any $\varepsilon>0$, there exist $\underline{m} \geq m_{0}$, $c>\sqrt{\varepsilon}$, and $B_{\varepsilon}^{ \pm} \subset C^{ \pm}(n ; c)$, such that for all $m>\underline{m}$,

$$
\nu_{h}\left(B_{\varepsilon}^{ \pm}\right)>0
$$

and for all $\omega \in B_{\varepsilon}^{ \pm}$,

$$
\forall \tau \in[0,1]: \quad \pm \omega(\tau) \geq \sqrt{\varepsilon} .
$$

Proof: Let us fix $p \in \mathbb{N} \backslash\{1\}, \alpha \in(0, p-1)$, and set

$$
\lambda_{\vartheta}(\omega)=\sup \left\{\frac{\left[\omega(\tau)-\omega\left(\tau^{\prime}\right)\right]^{2 p}}{\left|\tau-\tau^{\prime}\right|_{\text {per }}^{\alpha}}|0<| \tau-\left.\tau^{\prime}\right|_{\text {per }} \leq \vartheta\right\}, \quad \vartheta \in(0,1) .
$$

Then by the Garsia-Rodemich-Rumsey lemma, see e.g., pages 202, 203 in [6], one has from (56)

$$
\begin{aligned}
\int_{\mathcal{C}} \lambda_{\vartheta}(\omega) \nu_{h}(d \omega) & \leq \frac{2^{\alpha+6 p+\varsigma}}{p-\alpha-1}\left(1+\frac{2}{\alpha}\right) m^{-p} Q_{p} \vartheta^{p-\alpha} \\
& \stackrel{\text { def }}{=} m^{-p} Q_{p, \alpha} \vartheta^{p-\alpha},
\end{aligned}
$$


where $\varsigma>0$ is an absolute constant. Now we fix $n \geq 2$ and for $c>\sqrt{\varepsilon}$, define

$$
\begin{aligned}
A(c ; \varepsilon) & =\left\{\omega \in \mathcal{C} \mid \lambda_{1 / n}(\omega) \leq(c-\sqrt{\varepsilon})^{2 p} n^{\alpha}\right\}, \\
B_{\varepsilon}^{ \pm} & =A(c ; \varepsilon) \bigcap C^{ \pm}(n ; c) .
\end{aligned}
$$

Then for any $\tau \in[0,1]$, one can pick up $j / n$, such that

$$
|\omega(\tau)-\omega(j / n)| \leq\left[\lambda_{1 / n}(\omega)\right]^{1 / 2 p} n^{-\alpha / 2 p},
$$

which yields $\pm \omega(\tau) \geq \sqrt{\varepsilon}$ if $\omega \in B_{\varepsilon}^{ \pm}$. To estimate $\nu_{h}\left(B_{\varepsilon}^{ \pm}\right)$we proceed as follows. By (59) and (61), and by the Chebyshev inequality

$$
\begin{aligned}
\nu_{h}[\mathcal{C} \backslash A(c ; \varepsilon)] & \leq \frac{1}{(c-\sqrt{\varepsilon})^{2 p} n^{\alpha}} \int \lambda_{1 / n}(\omega) \nu_{h}(\mathrm{~d} \omega) \\
& \leq m^{-p} \cdot Q_{p, \alpha} /\left[n(c-\sqrt{\varepsilon})^{2}\right]^{p} .
\end{aligned}
$$

Now we set

$$
\sigma(n ; c)=\min \left\{\nu_{h}\left[C^{+}(n ; c)\right] ; \nu_{h}\left[C^{-}(n ; c)\right]\right\} .
$$

Thereby,

$$
\begin{aligned}
\nu_{h}\left(B_{\varepsilon}^{ \pm}\right) & \geq \sigma(n ; c)-\nu_{h}[\mathcal{C} \backslash A(c ; \varepsilon)] \\
& \geq \sigma(n ; c)-m^{-p} \cdot Q_{p, \alpha} /\left[n(c-\sqrt{\varepsilon})^{2}\right]^{p}
\end{aligned}
$$

which is positive for all

$$
m>\underline{m} \stackrel{\text { def }}{=} \max \left\{m_{0} ;\left[n(c-\sqrt{\varepsilon})^{2}\right]^{-1} \cdot\left[Q_{p, \alpha} / \gamma(n ; c)\right]^{1 / p}\right\} .
$$

Now we introduce

$$
\mathcal{C} \times \mathcal{C} \ni\left(\omega, \omega^{\prime}\right) \mapsto Y\left(\omega, \omega^{\prime}\right)=\int_{0}^{1} \omega(\tau) \omega^{\prime}(\tau) d \tau .
$$

Then, by (59)

$$
\forall \omega, \omega^{\prime} \in B_{\varepsilon}^{ \pm}: \quad Y\left(\omega, \omega^{\prime}\right) \geq \varepsilon .
$$

\subsection{The proof of the Lemmas}

Proof of Lemma 2.8; In the Euclidean approach, the periodic pressure (14) has the following representation, see (46), (47), and (54),

$$
p_{\Lambda}^{\mathrm{per}}(J, h)-p_{\Lambda}^{\mathrm{per}}(0, h)=|\Lambda|^{-1} \log \left\{\int_{\Omega_{\Lambda}} \exp \left[J Y_{\Lambda}\left(\omega_{\Lambda}\right)\right] \prod_{\ell \in \Lambda} \nu_{h}\left(d \omega_{\ell}\right)\right\},
$$

where

$$
Y_{\Lambda}\left(\omega_{\Lambda}\right)=\frac{1}{2} \sum_{\ell, \ell^{\prime} \in \Lambda,\left|\ell-\ell^{\prime}\right|_{\Lambda}=1} Y\left(\omega_{\ell}, \omega_{\ell^{\prime}}\right)
$$


and $Y$ being as in (64). For $\pm h \geq 0$, we get from (65), (64)

$$
\begin{aligned}
p_{\Lambda}^{\mathrm{per}}(J, h)-p_{\Lambda}^{\mathrm{per}}(0, h) & \geq|\Lambda|^{-1} \log \left\{\int_{\left(B_{\varepsilon}^{ \pm}\right)^{|\Lambda|}} \exp \left[J Y\left(\omega_{\Lambda}\right)\right] \prod_{\ell \in \Lambda} \nu_{h}\left(d \omega_{\ell}\right)\right\} \\
& \geq d J \varepsilon+\nu_{h}\left(B_{\varepsilon}^{ \pm}\right) .
\end{aligned}
$$

Now we fix $\varepsilon, c, n$, and $\alpha$. Then for a given $\delta>0$, we denote by $m_{*}$ the least value of $m \geq m_{0}$ for which the second line in (62) is $\geq \exp (-\delta)$. Thereafter, the latter estimate turns into (35).

Proof of Lemma 2.9: As $p_{\Lambda}^{\text {per }}$ is a convex function of $J$, we have

$$
\begin{aligned}
p_{\Lambda}^{\text {per }}(J, h)-p_{\Lambda}(0, h) & =\int_{0}^{J}\left(\frac{\partial}{\partial t} p_{\Lambda}^{\text {per }}(t, h)\right) d t \\
& \leq J \frac{\partial}{\partial J} p_{\Lambda}^{\text {per }}(J, h)
\end{aligned}
$$

Then by (65), 66), (64),

$$
\begin{aligned}
& \frac{\partial}{\partial J} p_{\Lambda}^{\mathrm{per}}(J, h)=\frac{1}{2|\Lambda|} \sum_{\ell, \ell^{\prime} \in \Lambda,\left|\ell-\ell^{\prime}\right|_{\Lambda}=1} \int_{\Omega_{\Lambda}} \omega_{\ell}(0) \omega_{\ell^{\prime}}(0) \mu_{\Lambda}^{\mathrm{per}}\left(\mathrm{d} \omega_{\Lambda}\right) \\
& \leq \frac{1}{4|\Lambda|} \sum_{\ell, \ell^{\prime} \in \Lambda,\left|\ell-\ell^{\prime}\right|_{\Lambda}=1} \int_{\Omega_{\Lambda}}\left\{\left[\omega_{\ell}(0)\right]^{2}+\left[\omega_{\ell^{\prime}}(0)\right]^{2}\right\} \mu_{\Lambda}^{\mathrm{per}}\left(\mathrm{d} \omega_{\Lambda}\right) \\
& \quad=\frac{1}{4|\Lambda|} \sum_{\ell, \ell^{\prime} \in \Lambda,\left|\ell-\ell^{\prime}\right|_{\Lambda}=1}\left\{\varrho_{\Lambda}^{\mathrm{per}}\left(q_{\ell}^{2}\right)+\varrho_{\Lambda}^{\mathrm{per}}\left(q_{\ell^{\prime}}^{2}\right)\right\}=d \varrho_{\Lambda}^{\mathrm{per}}\left(q_{\ell}^{2}\right)
\end{aligned}
$$

since $\varrho_{\Lambda}^{\text {per }}\left(q_{\ell}^{2}\right)$ is independent of $\ell$. Here, c.f. (65),

$$
\begin{aligned}
\mu_{\Lambda}^{\mathrm{per}}\left(d \omega_{\Lambda}\right) & =\exp \left[F_{\Lambda}(J, h)+J Y_{\Lambda}\left(\omega_{\Lambda}\right)\right] \prod_{\ell \in \Lambda} \nu_{h}\left(d \omega_{\ell}\right) \\
F_{\Lambda}(J, h) & =|\Lambda|\left[p_{\Lambda}(0,0)-p_{\Lambda}^{\mathrm{per}}(J, h)\right]
\end{aligned}
$$

is the Euclidean local Gibbs measure which corresponds to the Hamiltonian (7). Thereby, we employ (68) in (67) and get (36).

\section{References}

[1] Albeverio, S., Kondratiev, Y. G., Kozitsky, Y., and Röckner, M.: Euclidean Gibbs States of Quantum Lattice Systems. Rev. Math. Physics. 14, 1-67 (2002).

[2] Albeverio, S., Kondratiev, Y. G., Kozitsky, Y., and Röckner, M.: Small Mass Implies Uniqueness of Gibbs States of a Quantum Crystal. Commun. Math. Phys. 241, 69-90 (2003). 
[3] Albeverio, S., Kondratiev, Y. G., Kozitsky, Y., and Röckner, M.: Quantum Stabilization in Anharmonic Crystals. Phys. Rev. Lett. 90, 170603-1-4 (2003).

[4] Albeverio, S., Kondratiev, Y. G., Pasurek, T., and Röckner, M.: Euclidean Gibbs Measures on Loop Spaces: Existence and a Priori Estiamtes. Ann. Probab. 32, 153-190 (2004).

[5] Barbulyak, V. S. and Kondratiev, Y. G.: The Quasiclassical Limit for the Schrödinger Operator and Phase Transitions in Quantum Statistical Physics. Func. Anal. Appl. 26(2), 61-64 (1992)

[6] Barlow, M. T. and Yor, M.: Semi-martingale Inequalities via the GarsiaRodemich-Rumsey Lemma, and Applications to Local Times, J. Func. Anal., 49, 198-229 (1982)

[7] Driessler, W., Landau, L., and Perez, F. J.: Estimates of Critical Lengths and Critical Temperatures for Classical and Quantum Lattice Systems, J. Stat. Phys. 20, 123-162 (1979)

[8] Dyson, F. J., Lieb, E. H., and Simon, B.: Phase transitions in quantum spin systems with isotropic and nonisotropic interactions, J. Stat. Phys. 18, 335-383, (1978)

[9] Fröhlich, J., Simon, J., and Spencer, T.: Infrared Bounds, Phase Transitions and Continuous Symmetry Breaking, Commun. Math. Phys. 50, 79-85 (1976)

[10] Kondratiev, Ju. G.: Phase Transitions in Quantum Models of Ferroelectrics, pp. 465-475 in Stochastic Processes, Physics, and Geometry II, World Scientific, Singapore New Jersey (1994)

[11] Kozitsky, Y. and Pasurek, T.: Euclidean Gibbs Measures of Quantum Anharmonic Crystals. BiBoS Preprint 05-05-180 (2005)

[12] Pastur, L. A. and Khoruzhenko, B. A.: Phase Transitions in Quantum Models of Rotators and Ferroelectrics, Theoret. Math.Phys. 73, 111- 124 (1987)

[13] Simon, B.: The $P(\varphi)_{2}$ Euclidean (Quantum) Field Theory, Princeton Univ. Press, Princeton (1974)

[14] Simon, B.: Functional Integration and Quantum Physics, Academic Press, New York San Francisco London (1979)

[15] Simon, B.: The Statistical Mechanics of Lattice Gases, I., Princeton University Press, Princeton, New Jersey (1993)

[16] Stamenković, S.: Unified Model Description of Order-Disorder and Displacive Structural Phase Transitions. Condens. Matter Phys., 1(14), 257309 (1998) 